\title{
Modelling and refinement of hydrogen atoms: new developments in the CCP4 suite
}

\author{
L. Catapano ${ }^{1,2}$, R. A. Steiner ${ }^{1, *}$, G.N. Murshudov ${ }^{2, *}$ \\ ${ }^{1}$ Randall Centre for Cell and Molecular Biophysics, King's College London, London SE1 1UL, United Kingdom \\ ${ }^{2}$ MRC Laboratory of Molecular Biology, Francis Crick Avenue, Cambridge CB2 0QH, United Kingdom \\ *roberto.steiner@kcl.ac.uk and garib@mrc-lmb.cam.ac.uk
}

Hydrogen $(\mathrm{H})$ atoms often play essential roles in enzymatic reactions as they are responsible for the reversible protonation of active site residues and for the organisation of the solvent network. More generally, $\mathrm{H}$ atoms are also necessary for the establishment of $\mathrm{H}-$ bonds which, in turn, stabilise interactions between macromolecules and between macromolecules and their ligands. Although $\mathrm{H}$ atoms represent a large fraction of the total atomic content of macromolecules their direct visualisation is not straightforward. Even at (sub-)atomic resolution $(<1.2 \AA)$, X-ray macromolecular crystallography (MX), the most common technique for structural determination, affords the localisation of only a small percentage of $\mathrm{H}$ atoms as their contribution to the total scattering is minimal owing to their low electron content. Differently from MX, neutron macromolecular crystallography (NMX) relies on the interaction between neutrons and atomic nuclei. With this technique the visualisation of $\mathrm{H}$ atoms (typically in the form of deuterons, D) is possible even at modest resolution (2.0-2.5 $\AA$ ), making it an ideal complementary tool to MX when $\mathrm{H}$ localisation is deemed important.

Whilst NMX maps show the nuclear positions of $\mathrm{H}$ atoms, MX maps indicate the positions of valence-electron density for $\mathrm{H}$ atoms shifted along their bond vector. Cryo-electron microscopy (cryo-EM) and electron diffraction experiments inform on both nuclear and electron localisation of $\mathrm{H}$ atoms. This project aims at modelling and refining $\mathrm{H}$ atoms by using different experimental data (cryo-EM, NMX and electron diffraction) integrated in a common framework, to provide new insights in biological processes such as enzyme mechanisms. New features in the crystallographic refinement package REFMAC5 [1], one of the flagships of the scientific CCP4 computational suite, have been developed and will be presented. The CCP4 Monomer Library [2], typically used as the source for prior chemical information in REFMAC5 and other programs such as COOT [3], has been recently implemented for more accurate $\mathrm{H}$ atom positions derived from neutron data analysis [4] and quantum mechanics (QM) calculations. Recent developments in REFMAC5 and tools for the refinement of structural models obtained by neutron diffraction data will be presented.

[1] Kovalevskiy, O., Nicholls, R. A., Long, F., Carlon, A. \& Murshudov, G. N. (2018). Acta Cryst. D74, 215.

[2] Vagin, A. A., Steiner, R. A., Lebedev, A. A., Potterton, L., McNicholas, S., Long, F. \& Murshudov, G. N. (2004). Acta Cryst. D60, 2184.

[3] Emsley, P., Lohkamp, B., Scott, W. G. \& Cowtan, K. (2010). Acta Cryst. D66, 486.

[4] Allen, F. H. \& Bruno, I. J. (2010). Acta Cryst. B66, 380.

Keywords: neutron crystallography, macromolecular refinement; macromolecular crystallography; CCP4 